\title{
Designing Grid Structures Using Asymptotic Curve Networks
}

\author{
Eike Schling ${ }^{1(\bowtie)}\left(\mathbb{D}\right.$, Denis Hitrec ${ }^{2}$, and Rainer Barthel ${ }^{1}$ \\ ${ }^{1}$ Technical University of Munich, Arcisstr. 21, 80333 München, Germany \\ eike.schling@tum.de \\ ${ }^{2}$ University of Ljubljana, Zoisova Cesta 12, 1000 Ljubljana, Slovenia
}

\begin{abstract}
Doubly-curved grid structures pose great challenges in respect to planning and construction. Their realization often requires the fabrication of many unique and geometrically-complex building parts. One strategy to simplify the fabrication process is the elastic deformation of components to construct curved structures from straight elements. In this paper, we present a method to design doubly-curved grid structures with exclusively orthogonal joints from flat and straight strips of timber or steel. The strips are oriented upright on the underlying surface, hence normal loads can be transferred via bending around their strong axis. This is made possible by using asymptotic curve networks on minimal surfaces. We present the geometric and structural fundamentals and describe the digital design method including specific challenges of network and strip geometry. We illustrate possible design implementations and present a case study using a periodic minimal surface. Subsequently, we construct two prototypes: one timber and one in steel, documenting bespoke solutions for fabrication, detailing and assembly. This includes an elastic erection process, by which a flat grid is transformed into the spatial geometry. We conclude by discussing potential and challenges of this methods, as well as highlighting ongoing research in façade development and structural simulation.
\end{abstract}

Keywords: Asymptotic curves • Minimal surfaces · Elastic deformation · Gridshells

\section{Introduction}

There have been a number of publications and design strategies aiming to simplify the fabrication and construction process of doubly-curved grid structures. Therein, we can distinguish between discrete and smooth segmentations (Pottmann et al. 2015). One strategy to achieve affordable smoothly curved structures relies on the elastic deformation of its building components in order to achieve a desired curvilinear geometry from straight or flat elements (Lienhard 2014). Consequently, there is a strong interest in the modelling and segmentation of components that can be unrolled into a flat state, such as developable surfaces (Tang et al. 2016a). Recent publications have given a valuable overview on three specific curve types-geodesic curves, principal curvature lines, and asymptotic curves (Fig. 3) - that show great potential to be modelled as 


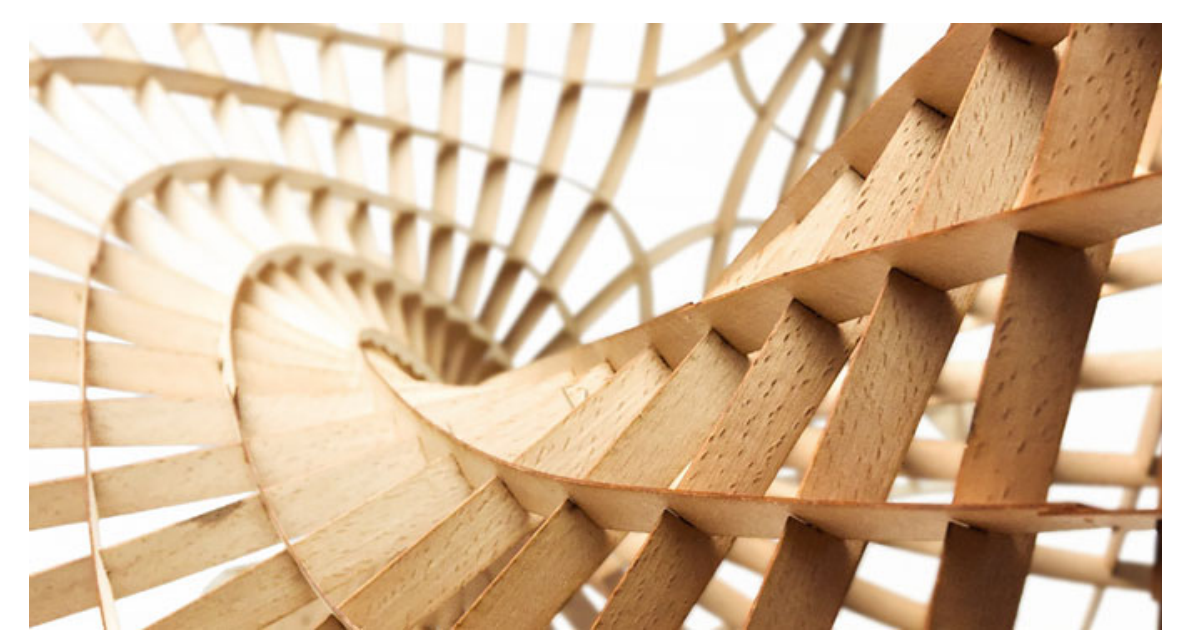

Fig. 1. Grid structure based on asymptotic curves: The model is built from straight strips of beech veneer. All joints are orthogonal. Image Denis Hitrec

developable strips (Tang et al. 2016a). Both geodesic curves and principle curvature lines have been successfully used for this purpose in architectural projects (Schiftner et al. 2012). However, there have been no applications of asymptotic curves for load-bearing structures. This is astounding, as asymptotic curves are the only type which are able to combine the benefits of straight unrolling and orthogonal nodes (Fig. 1).

In this paper we present a method to design strained grid structures along asymptotic curves to benefit from a high degree of simplification in fabrication and construction. They can be constructed from straight strips orientated normal to the underlying surface. This allows for an elastic assembly via their weak axis, and a local transfer of normal loads via their strong axis. Furthermore, the strips form a doubly-curved network, enabling a global load transfer as a shell structure (Schling and Barthel 2017).

In Section "Fundamentals", we describe the geometric theory of curvature and related curve networks, and present the strained gridshells of Frei Otto, as our primary reference for construction. In Section "Design Method" we introduce our computational design method of modelling minimal surfaces, asymptotic curves and networks, and the related strip-geometry. In Section "Design Implementation", we illustrate our method in a typological overview of shapes and implement it in a design study of a pavilion. In Section "Construction", we discuss the fabrication, construction details and assembly by means of two prototypes, in timber and steel, and give insights into the load-bearing behavior. The results are summarized in Section "Results". We conclude in Section "Conclusion", by highlighting challenges of this method, and suggesting future investigations on structural simulation and prototype development. 


\section{Fundamentals}

\section{Geometry}

Curvature. The curvature of a curve is the inverse of the curvature radius. It is measured at the osculating circle of any point along the curve (Fig. 2 left).

To determine the curvature of a surface, we intersect it with orthogonal planes through the normal vector. The two section-curves with the highest and lowest curvature are perpendicular to each other and indicate the principle curvature directions (Fig. 2 middle). The two principle curvatures $k_{1}$ and $k_{2}$ are used to calculate the Gaussian curvature $\left(G=k_{1} \times k_{2}\right)$ and mean curvature $\left(H=\left(k_{1}+k_{2}\right) / 2\right)$. If $k_{1}$ and $k_{2}$ have opposite orientations, i.e. their osculating circles lie on opposite sides of the surface, then the Gaussian curvature has a negative value. This surface-region is called anticlastic or hyperbolic. If $k_{1}$ and $k_{2}$ have opposite orientations and the same absolute value, then the mean curvature is zero. Surfaces with a constant zero mean curvature are called minimal surfaces. They are a special type of anticlastic surfaces that can be found in nature in the shape of soap films.

To measure the curvature of a curve on a surface, we can combine the information of direction (native to the curve) and orientation (native to the surface) to generate a coordinate system called the Darboux frame (Fig. 2 right). This frame consists of the normal vector $\mathbf{z}$, the tangent vector $\mathbf{x}$ and their cross-product, the tangent-normal vector y. When moving the Darboux frame along the surface-curve, the velocity of rotation around all three axes can be measured. These three curvature types are called the geodesic curvature $k_{g}$ (around $\mathbf{z}$ ), the geodesic torsion $t_{g}$ (around $\mathbf{x}$ ), and the normal curvature $k_{n}$ (around y) (Tang et al. 2016b).

Curve networks. Certain paths on a surface may avoid one of these three curvatures (Fig. 3). These specific curves hold great potential to simplify the fabrication and construction of curved grid structures (Schling and Barthel 2017). Geodesic curves have a vanishing geodesic curvature. They can be constructed from straight, planar strips tangential to the surface. Principle curvature lines have a vanishing geodesic torsion-there is no twisting of the respective structural element. They can be

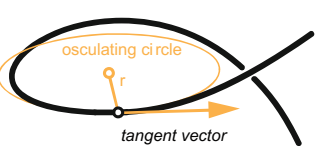

curvature $k=1 / r$

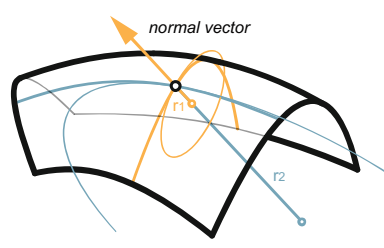

Gaussian curvature $\mathrm{K}=\mathrm{k}_{1} \times \mathrm{k}_{2}$ mean curvature $\quad \mathrm{H}=\left(\mathrm{k}_{1}+\mathrm{k}_{2}\right) / 2$

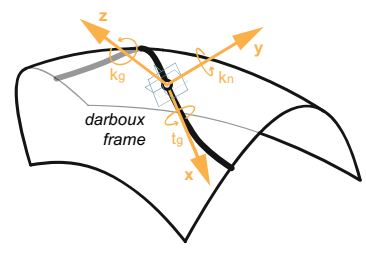

$\mathrm{k}_{\mathrm{n}}=$ normal curvature $\mathrm{kg}_{\mathrm{g}}=$ geodesic curvatu re $\mathrm{tg}_{\mathrm{g}}=$ geodesic torsion

Fig. 2. Definitions of curvature. Left Curvature of a curve is measured through the osculating circle. Middle The curvature of a surface is calculated through the two principle curvatures $k_{1}$ and $k_{2}$ as Gaussian or mean curvature. Right There are three curvatures of a curve on a surface. Geodesic curvature $k_{g}$, geodesic torsion $t_{g}$ and normal curvature $k_{n}$ 

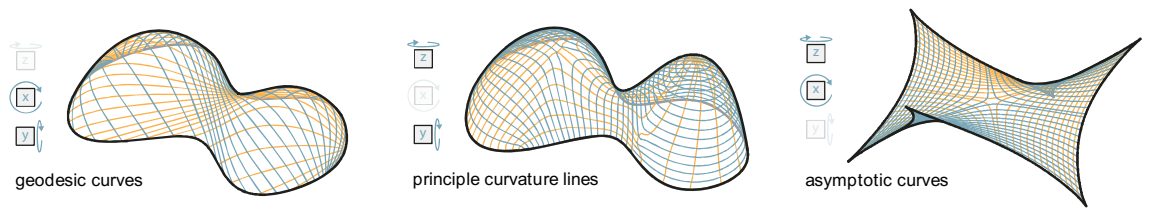

Fig. 3. Surface-curves have three curvatures: geodesic curvature (z), geodesic torsion (x), and normal curvature (y). For each of them, if avoided, a related curve type exists: geodesic curves, principle curvature lines and asymptotic curves

fabricated from curved, planar strips, and bent only around their weak axis. Their two families intersect at $90^{\circ}$. Asymptotic curves have a vanishing normal curvature, and thus only exist on anticlastic surface-regions. Asymptotic curves combine both geometric benefits: They can be formed from straight, planar strips perpendicular to the surface. On minimal surfaces, their two families intersect at $90^{\circ}$ and bisect principle curvature lines.

The design of related curve-networks is not trivial. Geodesic curve networks tend to vary in density and intersection angles. The designer may choose their start and end point but not their path. Both principle curvature networks and asymptotic curve networks consist of two families of curves that follow a direction field. The designer can only pick a starting point, but cannot alter their path. If the surface is locally planar (or spherical), the quadrilateral network forms a singularity with higher or lower valence.

\section{Construction}

Our construction method (Section "Construction") is based on the strained timber gridshells of Frei Otto (Fig. 4). This paradigm utilizes elastic deformation to create a doubly-curved lattice structure from straight wooden laths.

Otto's curve network is subject to all three types of curvature (as described in Section "Geometry"). Thus, the laths need to be bent and twisted in all directions. The

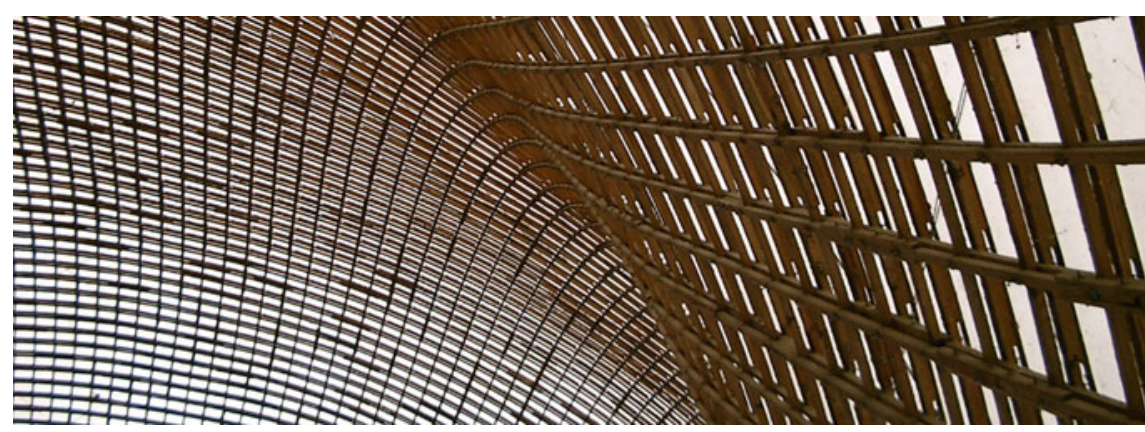

Fig. 4. Multihalle Mannheim by Frei Otto, 1975. This strained timber gridshell is formed from elastically-bent timber laths. Image Rainer Barthel 
doubly-symmetrical profiles must be appropriately flexible in bending and torsion (Schling and Barthel 2017). Otto's lattice grid is assembled flat and subsequently erected into a doubly-curved inverted funicular shape. Once the final geometry is reached, the quadrilateral network is fixed along the edges and braced with diagonal cables (Adriaenssens and Glisic 2013).

\section{Design Method}

Minimal surface. A minimal surface is the surface of minimal area between any given boundaries. In nature such shapes result from an equilibrium of homogeneous tension, e.g. in a soap film. The accurate form is found digitally in an iterative process by either minimizing the area of a mesh, or finding the shape of equilibrium for an isotropic pre-stress field.

Various tools are capable of performing such optimization on meshes, with varying degrees of precision and speed (Surface Evolver, Kangaroo-SoapFilm, Millipede, etc.). They are commonly based on a method by Pinkall and Polthier (2013).

The Rhino-plugin TeDa (Chair of Structural Analysis, TUM) provides a tool to model minimal surfaces as NURBS, based on isotropic pre-stress fields (Philipp et al. 2016).

Certain minimal surfaces can be modelled via their mathematical definition. This is especially helpful as a reference when testing the accuracy of other tools.

Asymptotic curves. Geometrically, the local direction of an asymptotic curve can be found by intersecting the surface with its own tangent plane.

We developed a custom VBScript for Grasshopper/Rhino to trace asymptotic curves on NURBS-surface using differential geometry. For this, the values and directions of the principal curvature $\left(k_{1}, k_{2}\right)$ are retrieved at any point on the surface. With this information, we can calculate the normal curvature $k_{n}$ for any other direction with deviation-angle $\alpha$ (Fig. 6 left) (Pottmann et al. 2007, p. 490).

$$
k_{n}(\alpha)=k_{1}(\cos \alpha)^{2}+k_{2}(\sin \alpha)^{2} .
$$

To find the asymptotic directions, the normal curvature must be zero, $k_{n}(\alpha)=0$. Solving for $\alpha$ results in:

$$
\alpha=2 \pi-2 \tan ^{-1} \sqrt{\frac{2 \sqrt{k_{2}\left(k_{2}-k_{1}\right)}+k_{1}-2 k_{2}}{k_{1}}} .
$$

By iteratively walking along this asymptotic direction and calculating a new $\alpha$ at every step, we can draw an asymptotic curve on any anticlastic surface. Our algorithm uses the Runge-Kutta method to average out inaccuracies due to step size. On minimal surfaces, the deviation angle $\alpha$ is always $45^{\circ}$ (due to the bisecting property of asymptotic curves and principle curvature lines).

In the case of meshes, we use EvoluteTools to find the asymptotic curves. Both EvoluteTools and our VBScript were checked for accuracy by comparing their results 
with a parametrically defined asymptotic network on an Enneper minimal surface. Depending on the quality of the mesh or NURBS surface and the step size, a sufficient accuracy for design and planning can be achieved.

Network design. When designing an asymptotic curve network, we take advantage of the bisecting property between asymptotic curves and principle curvature lines (Pottmann et al. 2007, p. 648). By alternately drawing each curve and using their intersections as new starting point, we can create an "isothermal" web with nearly quadratic cells (Fig. 5) (Sechelmann et al. 2012). Combining these two networks allows us to benefit from both their geometric properties simultaneously for substructure and façade, allowing for cladding solutions with tangential developable strips or planar quads (Pottmann et al. 2007, p. 680).

Strip geometry and fabrication. A developable, i.e., singly-curved, surface-strip is defined by straight rulings. If this strip is orthogonal to the reference surface and follows a given surface-curve, the rulings are enveloped by all planes that contain the normal vector $\mathbf{z}$ and tangent vector $\mathbf{x}$ of the Darboux frame (Fig. 6 left). The vector of these rulings $\mathbf{r}$ is calculated via the equation

$$
\mathbf{r}=k_{g} \mathbf{z}+t_{g} \mathbf{x}
$$

where $k_{g}$ is the geodesic curvature and $t_{g}$ is the geodesic torsion. For asymptotic curves, $k_{g}$ is measured simply via its osculating circles. To calculate $t_{g}$ we use the two principle curvatures (Tang et al. 2016b):

$$
t_{g}=\frac{1}{2}\left(k_{2}-k_{1}\right) \sin 2 \alpha,
$$

where $\alpha$ is constant $45^{\circ}$ on a minimal surface. The rulings of a developable strip along asymptotic curves are not necessarily perpendicular to the surface. Thus two orthogonal strips commonly have a curved intersection (Fig. 6 middle). In the worst case of a vanishing geodesic curvature, the rulings are parallel to the tangent vector, which makes modelling and construction of a developable surface-strip impossible.

In our method, the strip geometry is defined with the normal vectors $\mathbf{z}$, which allows for straight intersections and well-defined strip surfaces (Fig. 6 right). As a consequence, some twisting of the structural strips needs to be considered. This
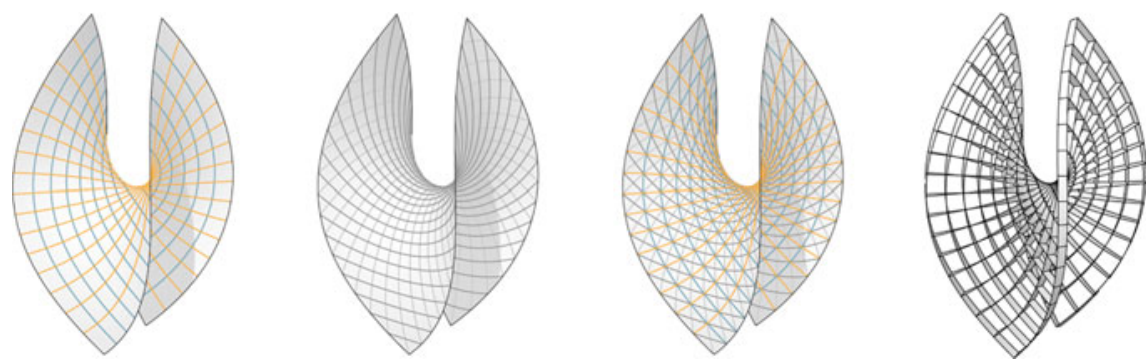

Fig. 5. Enneper surface with a asymptotic curves $\mathbf{b}$ principle curvature lines $\mathbf{c}$ web of both networks $\mathbf{d}$ strip model of the asymptotic network 

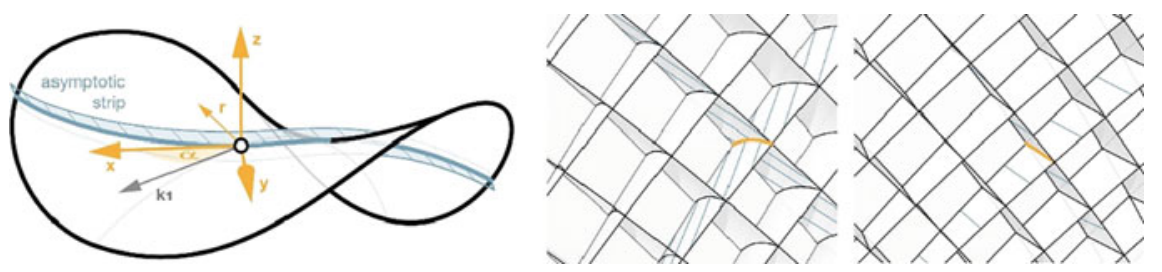

Fig. 6. Left A developable strip along an asymptotic curve and orthogonal to the surface, is define by rulings $\mathrm{r}$, which are generally not parallel to the normal vector $\mathrm{z}$. Middle This results in curved intersections between the strips. Right In our method, the strips are defined by the normal vectors z, to ensure straight intersections. Consequently, the strips are not truly developable, but twisted

deviation from a truly developable strip is essential to realizing a simplified construction.

The node to node distance, measured along the asymptotic curves, is the only variable information needed to draw the flat and straight strips. They are cut flat and then bent and twisted into an asymptotic support structure.

\section{Design Implementation}

Design strategies. Even though minimal surfaces can only be designed through their boundaries, there is a wide range of possible shapes and applications. Examples shown in Fig. 7 display how varying boundary conditions influence the surface and asymptotic network. Boundary-curves may consist of straight lines (a), planar curves (d), or spatial curves (b). Straight or planar curves are likely to attract singularities (a, d). A well-integrated edge can be achieved by modelling a larger surface and "cookie-cutting" the desired boundary. A minimal surface can be defined by one (a, b), two (c), or multiple (d) closed boundary-curves. Symmetry properties can be used to create repetitive (c) and periodic (e) minimal surfaces. The Gaussian curvature of the design surface directly influences the geodesic torsion of asymptotic curves, the density of the network and the position of singularities. A well-balanced Gaussian curvature will produce a more homogenous network.

Case study. We applied this method in a design studio, Experimental Structures, to develop the concept for a research pavilion. The design shape is based on the periodic minimal surface, Schwarz D, which can be described within a simple boundary along six edges of a cube. This basic cell is repeatedly copied and rotated to form an infinitely repetitive surface. Finally, this surface is clipped with an inclined block to cut out the desired shape. The entire network is a repetition of the curve-segments within one-sixth of the initial cubic cell (Fig. 8).

Despite the high level of repetition, the pavilion displays a complex and sculptural shape. A model $(2 \times 2 \times 1 \mathrm{~m})$ was built from strips of beech veneer at a scale of 1:5 to verify the fabrication and assembly process (Fig. 9). 

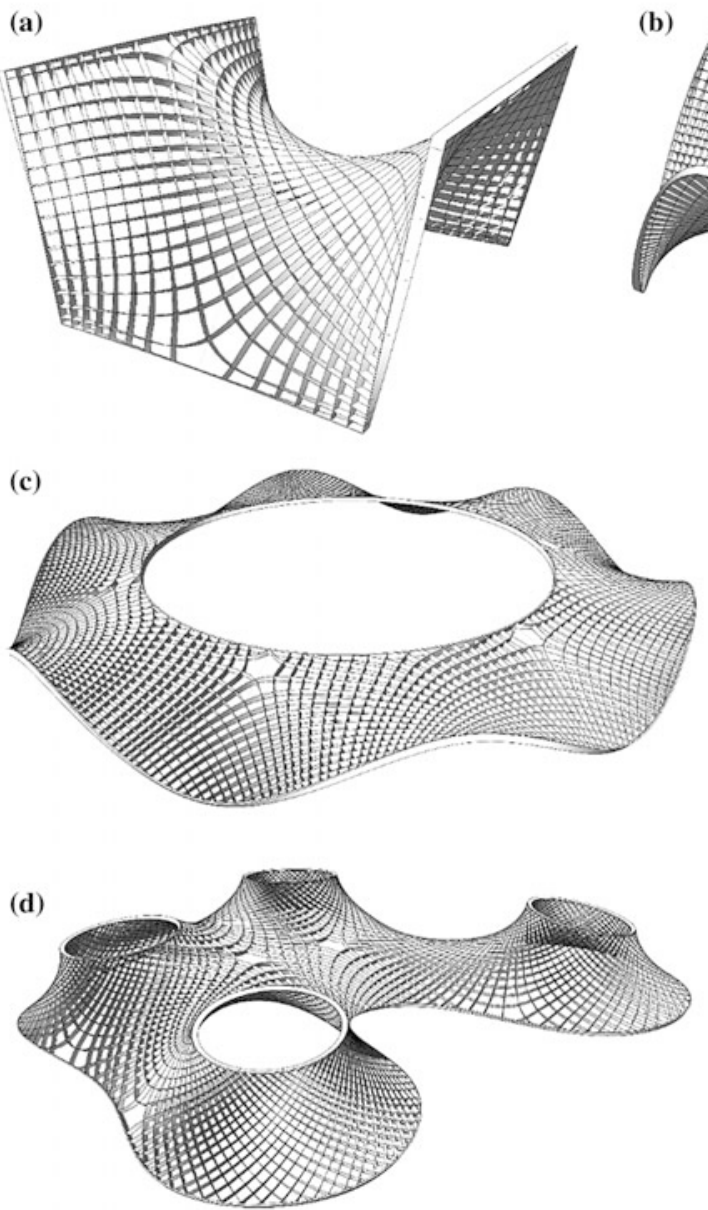

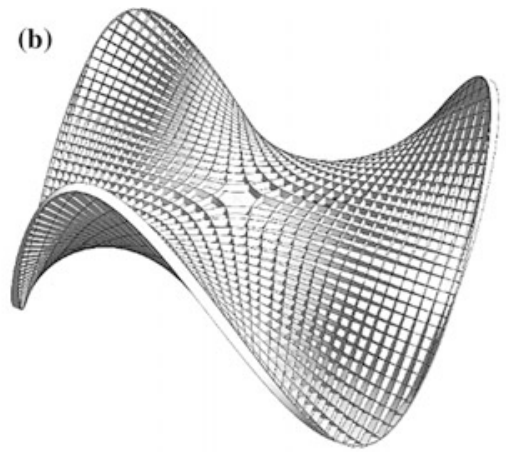

(e)

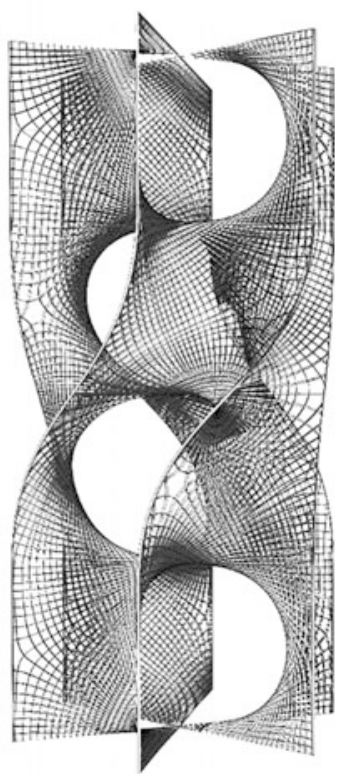

Fig. 7. Overview of asymptotic strip networks on minimal surfaces. a One polygonal boundary, creating a saddle shaped network with singularities appearing along the edges. b One spatially curved boundary, creating a surface with three high and low points and a network with central singularity. c Two boundary curves creating a rotational repetitive network with regular singularities. d Multiple boundaries creating a freely design minimal surface with four high points. e Variation of a singly-periodic Sherk's two minimal surface, with six interlinking boundaries 

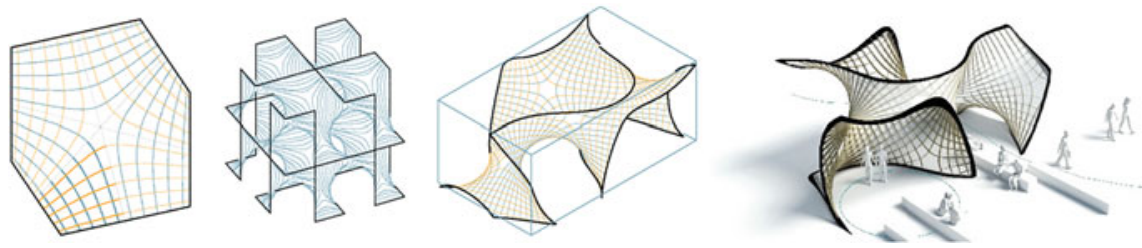

Fig. 8. Design implementation using a Schwarz D periodic minimal surface

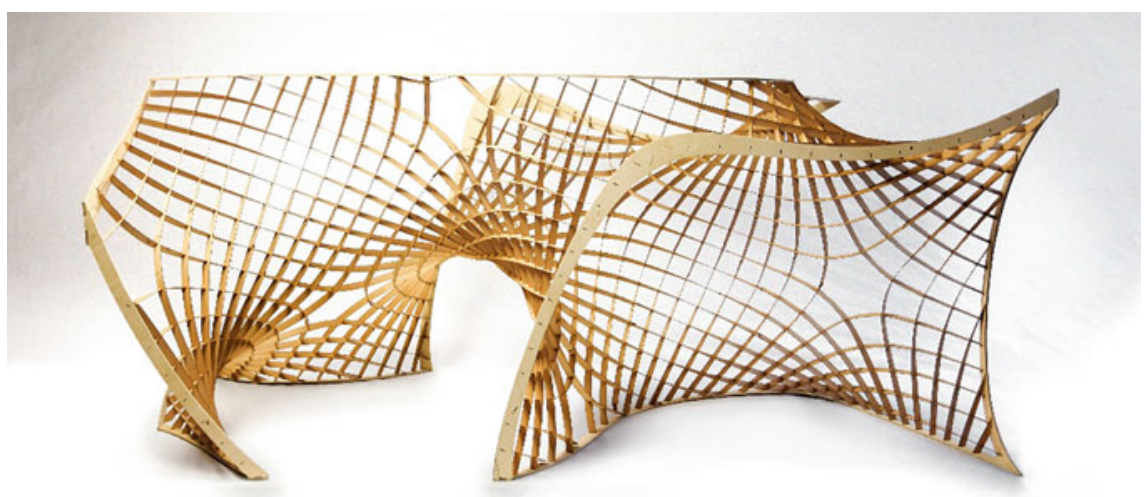

Fig. 9. Model of the design implementation. The structure is built from straight timber strips of beech veneer with exclusively orthogonal nodes. Image and model Denis Hitrec

\section{Construction}

Curvature and bending. Our construction process follows the reference of Frei Otto's strained gridshells (see Section "Construction"). The strips are fabricated flat and subsequently bent into their spatial geometry. As asymptotic curves admit no normal curvature, no bending in the strong axis of the strips is necessary during assembly. Due to the geodesic torsion, there is a certain amount of twisting of the lamellas. Additionally, the geodesic curvature results in bending around the z-axis.

When choosing the profiles, the section modulus and thickness need to be adjusted to the maximum twist and minimal bending radii to keep deformation elastic. At the same time, the profiles need to provide enough stiffness to resist buckling under compression loads. These opposing factors can be solved by introducing a second parallel layer of lamellas. Each layer is sufficiently slender to easily be bent and twisted into its target geometry. Once the final geometry is fixed, the two layers are connected with a shear block in regular intervals to increase the overall stiffness similar to a Vierendeel truss.

This technique was applied in the construction of two prototypes, in timber and steel, each with an approx. $4 \times 4 \mathrm{~m}$ span (Figs. 10 and 11).

Timber prototype-spatial construction. For the timber prototype, the two asymptotic directions were constructed on separate levels out of $4 \mathrm{~mm}$ thick poplar 

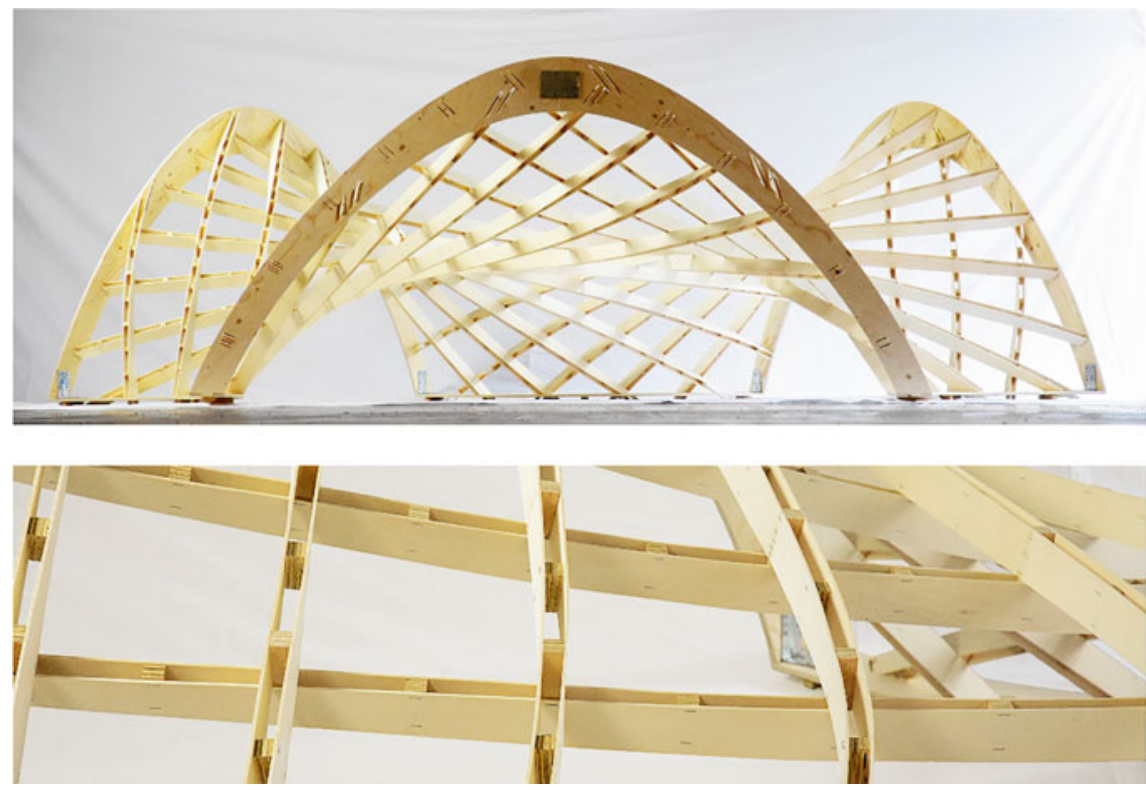

Fig. 10. Timber prototype. The lamellas are doubled and coupled to allow for low bending radii and high stiffness. Image Eike Schling

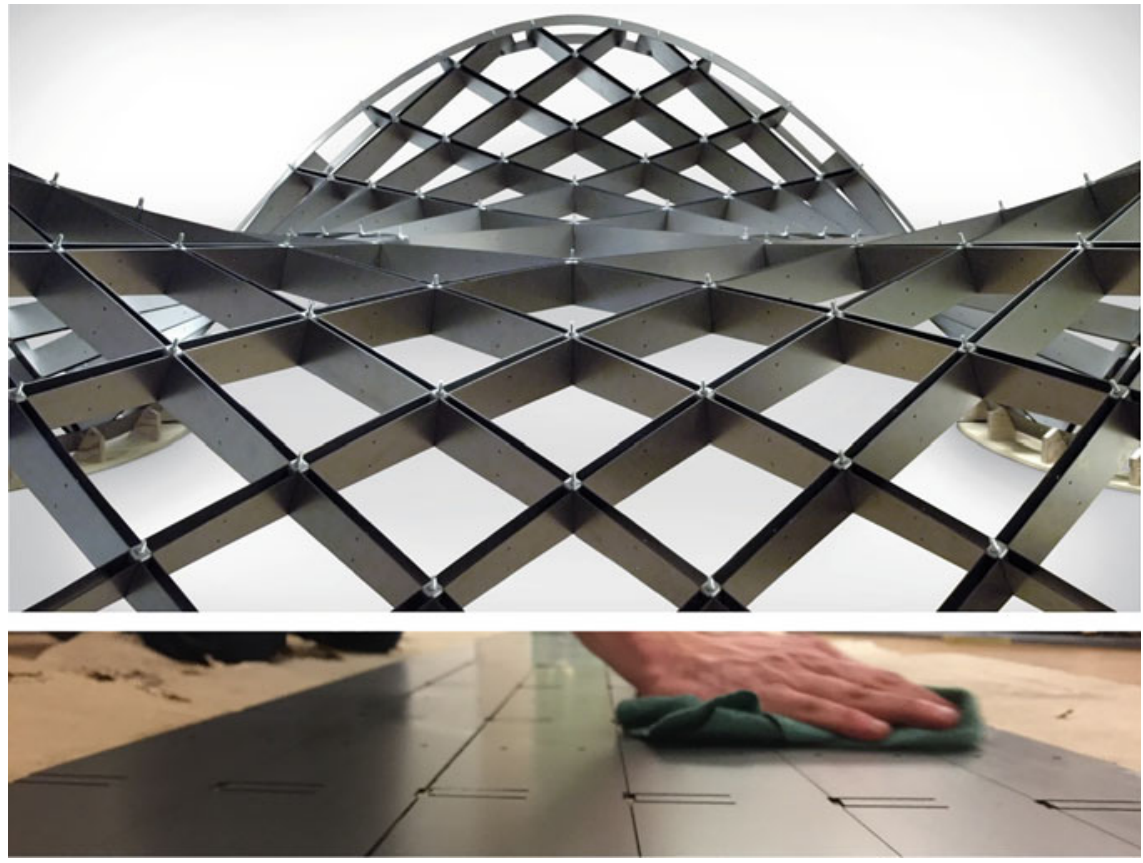

Fig. 11. Steel prototype. The straight lamellas are doubled and coupled to allow for low bending radii and high stiffness. Image Eike Schling 
plywood strips. This allowed for the use of continuous, uninterrupted profiles. The upper and lower level were connected with a square stud, enforcing the orthogonal intersection angle. This rigid connection could only be fitted if all elements were curved in their final geometry. Consequently, this prototype had to be erected spatially using framework and edge beams as temporary supports. The height of the edge profiles was determined by their intersection angle with the lamellas, creating a dominant arched frame.

Steel prototype-elastic erection. The steel prototype was built from straight, $1.5 \mathrm{~mm}$ steel strips. Both strip families interlock flush on one level. Therefore, the lamellas have a double slot at every intersection (Fig. 11). Due to a slot tolerance, the joints were able to rotate by up to $60^{\circ}$. This made it possible to assemble the grid flat on a hexagonal scaffolding. The structure was then "eased down" and "pushed up" simultaneously and thus transformed into its spatial geometry (Fig. 12) (Quinn and Gengnagel 2014). During the deformation process, a pair of orthogonal, star-shaped washers were tightened with a bolt at every node, enforcing the $90^{\circ}$ intersection angle.
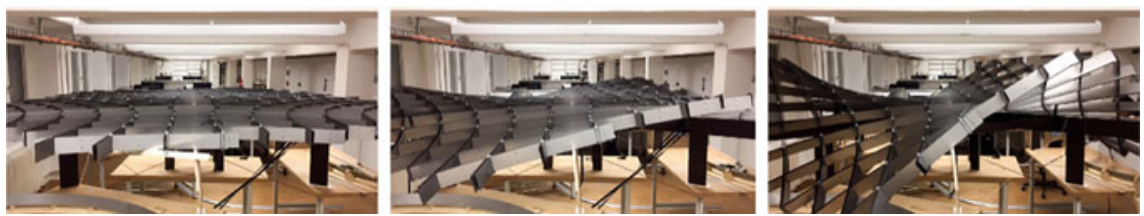

Fig. 12. Assembly process of the steel prototype, showing the elastic transformation from flat to curved geometry. Image Denis Hitrec

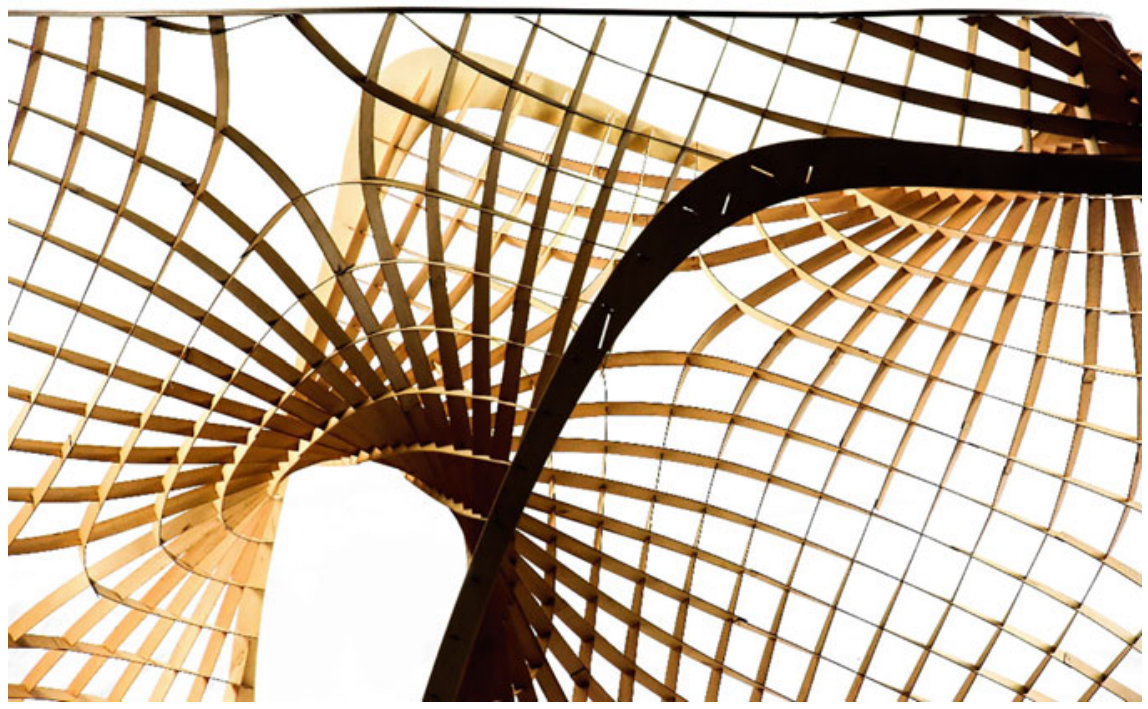

Fig. 13. Silhouette of the strip model from beech veneer 
Once the final geometry was reached, the edges were fitted as tangential strips on top and bottom. This edge locks the shape in its final geometry, generates stiffness and provides attachments for the future diagonal bracing and façade (Figs. 13, 14 and 15).

Structural behavior. The structural behavior of asymptotic grids is greatly dependent on the overall shape and support of the structure. Our initial investigations have observed a hybrid load-bearing behaviour of both a grillage and a gridshell (Figs. 16 and 17).

The strip-profiles are orientated normal to the underlying surface, allowing for a local transfer of normal loads through bending via their strong axis. This is especially helpful to account for the local planarity of asymptotic networks (due to their vanishing normal curvature) and to stabilize open edges.

On the other hand, the strips form a doubly-curved network, enabling a global load transfer as a gridshell (Schling and Barthel 2017). For this, the quadrilateral grid needs to be appropriately braced via diagonal cables. The edge configuration adds additional stiffness by creating triangular meshes.

The elastic erection process, results in residual stresses inside the curved grid elements. Additional compression forces, originating from a membrane load-bearing behavior, increase the bending moment around the weak axis of these curved elements. The strategy of doubling and coupling lamellas (see Section "Curvature and bending") is therefore essential to control local bucking.

Finally, it needs to be said, that the principle stress trajectories of a shell constitute the optimal orientation for compression and tension elements in a respective grid structure. In our method, however, we choose to follow a geometrically optimized orientation along the asymptotic directions.

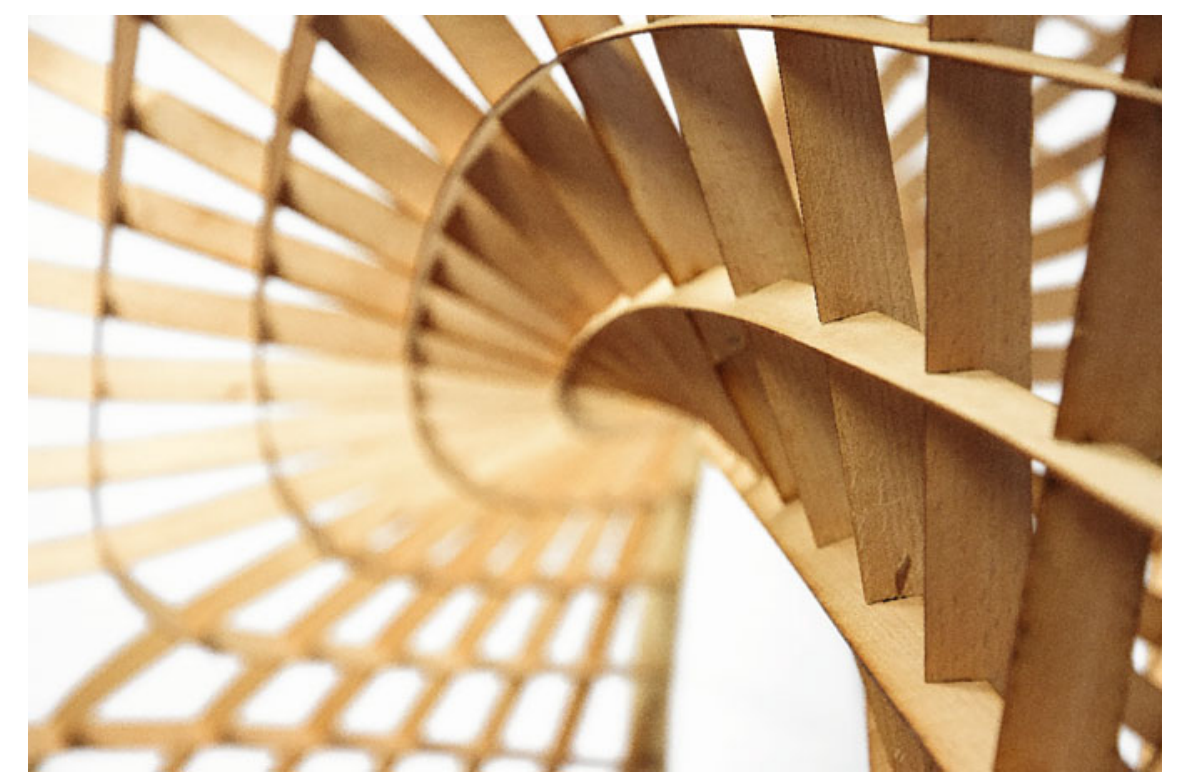

Fig. 14. Close-up of the strip model from beech veneer 


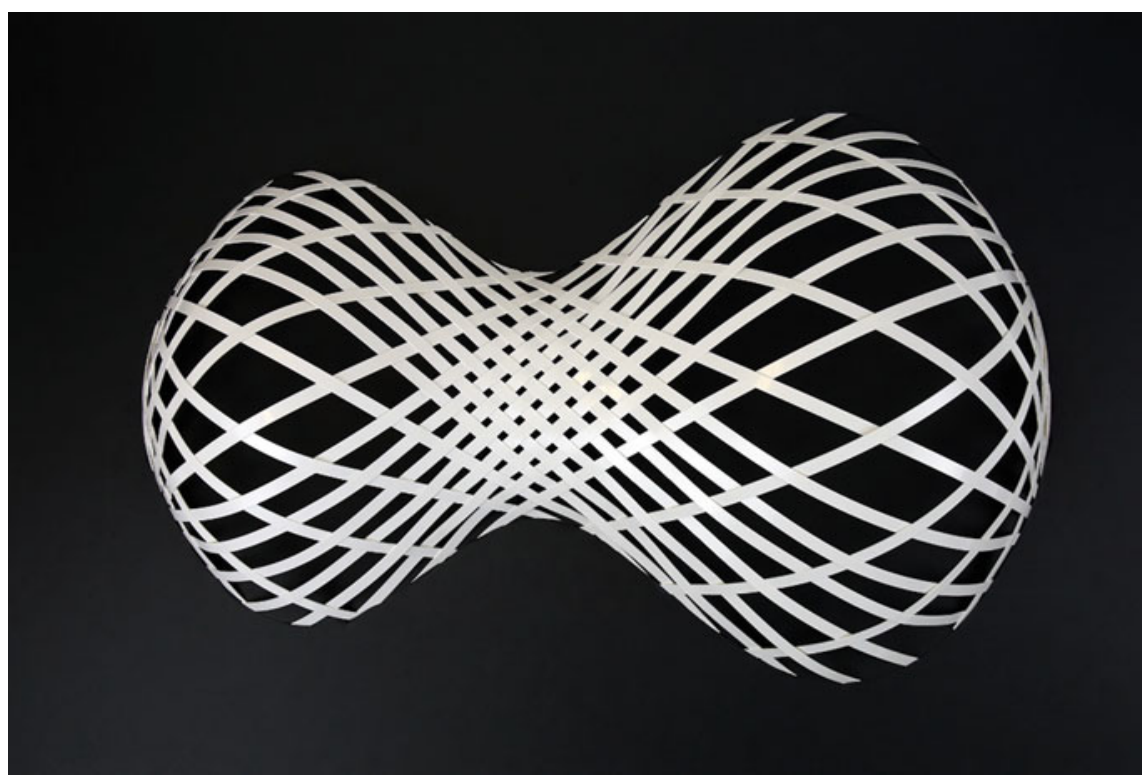

Fig. 15. Strip model of a geodesic curve network

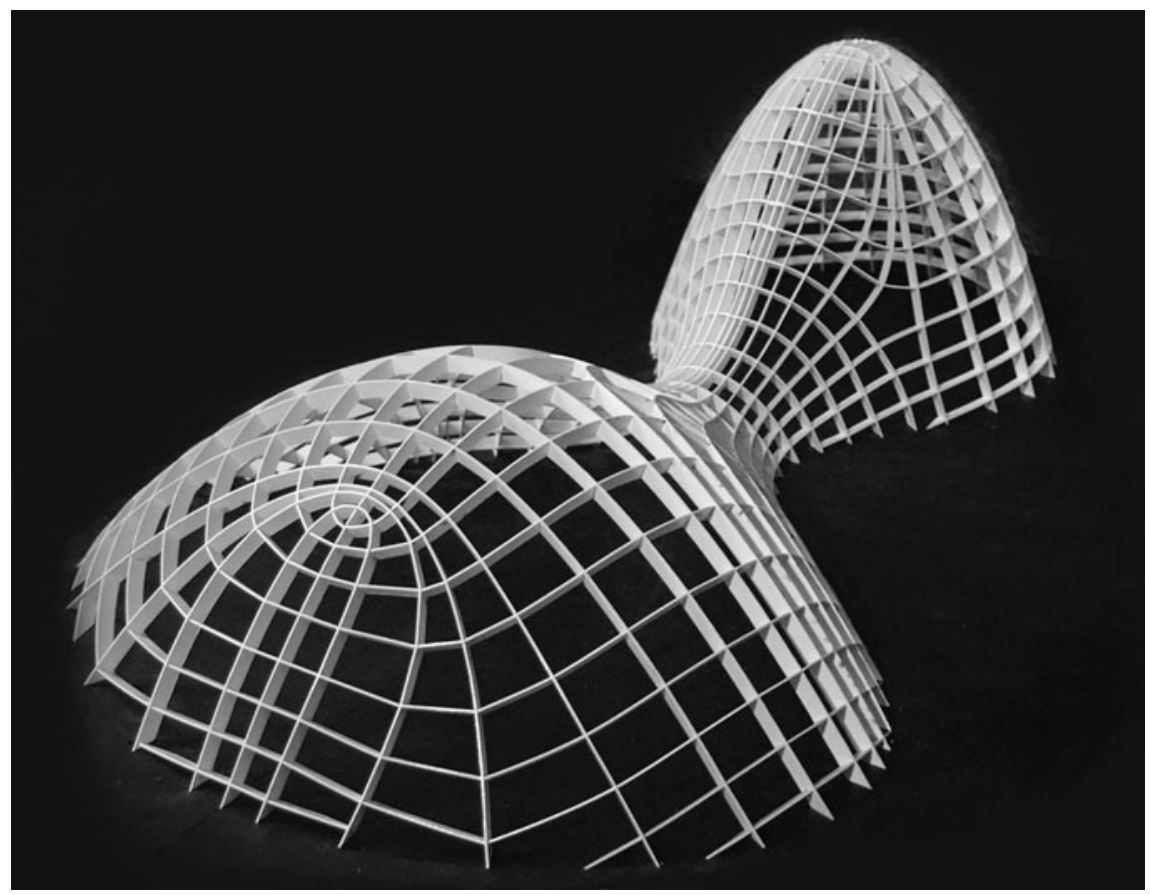

Fig. 16. Strip model of a principle curvature line network 


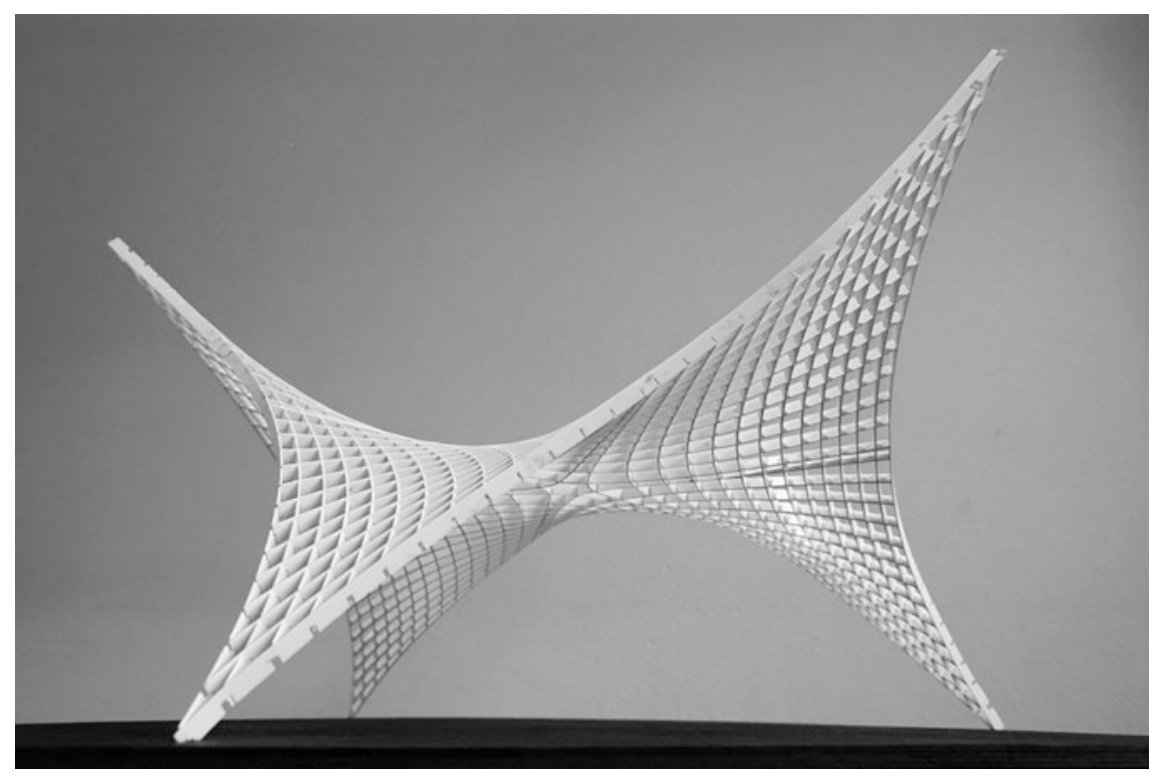

Fig. 17. Strip model of an asymptotic curve network

\section{Results}

We compared the geometric properties of three specific curve networks: geodesic curves, principle curvature lines and asymptotic curves.

We identified that only asymptotic curves are able to combine the benefits of straight unrolling and orthogonal nodes. They can be formed from straight strips perpendicular to the underlying anticlastic surface. This way, they resist loads normal to the surface by bending in their strong axis. On minimal surfaces, asymptotic curves intersect at $90^{\circ}$, which allows the use of identical nodes throughout the structure. The bisecting property with principle curvature networks offers further geometric advantages for the façade.

We developed a custom VBScript that can trace asymptotic curves on anticlastic surfaces with sufficient accuracy for design and construction. A wide spectrum of design solutions was visualized in a typological overview of strip networks and implemented in a case study for a pavilion.

We model the strip-network along the normal vectors, to ensure a well-defined geometry and simplified construction with identical and straight intersections, thus deviating from a true developable geometry. This measure results in some twisting of the profiles.

Both twisting (geodesic torsion) and bending (geodesic curvature) have to be considered when choosing profiles for construction. We have presented a strategy of doubling and coupling the bent structural elements to achieve sufficient stiffness of the final grid. We demonstrated an elastic erection process enabling assembly in a flat state and a subsequent transformation into a spatially-curved geometry. The findings were 
verified in the realization of two prototypes: One in timber and one in steel, each with a span of $4 \times 4 \mathrm{~m}$. The structural behavior of asymptotic grids was discussed along the basis of both a grillage and a gridshell.

\section{Conclusion}

An analytical approach to both geometry and material properties is required to achieve a symbiosis of form, structure and fabrication. Even though the design freedom is limited to the choice of boundary curves, there are a wide range of design solutions. Additionally, asymptotic networks offer an individual aesthetic quality. Our design method may be applied in all scales from furniture design to stadium roofs. Structurally, asymptotic gridshells show great potential, as they combine the benefits of upright sections with a doubly-curved grid. Hence, loads can be transferred locally via bending, and globally as a shell structure.

We are continuing to investigate the structural behavior of asymptotic structures on the basis of a grillage and a shell, comparing grid orientations, shapes and supports. Simultaneously, we are developing a workflow to compute the residual stress of the initial bending and torsion through the local geodesic curvature and geodesic torsion, without simulating the construction process. Another ongoing development is the implementation of constructive details: This includes cable bracing and façade systems using planar quads, developable façade strips and membranes.

Acknowledgements. This paper is part of the research project, Repetitive Grid Structures, funded by the Leonhard-Lorenz-Stiftung and the Research Lab of the Department of Architecture, TUM. Both the geometric background as well as the digital workflow were developed in close collaboration with the Department of Applied Geometry and the Center for Geometry and Computational Design, Prof. Helmut Pottmann, TU Wien. The plugin TeDa was provided and supported by Anna Bauer and Bendikt Phillipp from the Chair of Structural Analysis, TUM. Further support in MESH modelling was granted by Alexander Schiftner and the Evolute GmbH in Perchtoldsdorf, Austria. We would like to thank Matthias Müller, locksmith at the Technisches Zentrum, TUM, as well as Thomas Brandl and Harry Siebendritt of the Brandl Metallbau $\mathrm{GmbH \& Co.} \mathrm{KG} \mathrm{in} \mathrm{Eitensheim} \mathrm{for} \mathrm{their} \mathrm{extensive} \mathrm{support} \mathrm{in} \mathrm{steel} \mathrm{fabrication.}$

\section{References}

Adriaenssens, S., Glisic, B.: Multihalle Mannheim. Princeton University, Department of Civil and Environmental Engineering. http://shells.princeton.edu/Mann1.html (2013). Accessed 9 May 2017

Lienhard, J.: Bending-Active Structures. Form-Finding Strategies Using Elastic Deformation in Static and Kenetic Systems and the Structural Potentials Therein. ITKE, Stuttgart (2014)

Philipp, B., Breitenberger, M., Dàuria, I., Wüchner, R., Bletzinger, K.-U.: Integrated design and analysis of structural membranes using isogeometric B-rep analysis. In: Computer Methods in Applied Mechanics and Engineering, vol. 303, pp. 312-340 (2016)

Pinkall, U., Polthier, K.: Computing discrete minimal surfaces and their conjugates. Exp. Math. 2 (1), 15-36 (2013) 
Pottmann, H., Asperl, A., Hofer, M., Kilian, A.: Architectural Geometry. Bentley Institute Press, Exton (2007)

Pottmann, H., Eigensatz, M., Vaxman, A., Wallner, J.: Architectural geometry. In: Computers and Graphics. http://www.geometrie.tuwien.ac.at/geom/fg4/sn/2015/ag/ag.pdf (2015). Accessed 9 May 2017

Quinn, G., Gengnagel, C.: A review of elastic grid shells, their erection methods and the potential use of pneumatic formwork. In: De Temmerman, N., Brebbia, C.A. (eds.) MARAS 2014. Ostend, WIT Press, Southampton, pp. 129-143 (2014)

Schiftner, A., Leduc, N., Bompas, P., Baldassini, N., Eigensatz, M.: Architectural geometry from research to practice: the eiffel tower pavilions. In: Hesselgren, L., et al. (eds.) Advances in Architectural Geometry, pp. 213-228. Springer, New York (2012)

Schling, E., Barthel, R.: Experimental studies on the construction of doubly curved structures. In: DETAIL structure 01/17, Munich, Institut für Internationale Architektur-Dokumentation, pp. 52-56 (2017)

Sechelmann, S., Rörig, T., Bobenko, A.: Quasiisothermic mesh layout. In: Hesselgren, L., et al. (eds.) Advances in Architectural Geometry, pp. 243-258. Springer, New York (2012)

Tang, C., Bo, P., Wallner, J., Pottmann, H.: Interactive design of developable surfaces. In: ACM Transactions on Graphics, vol. 35, No. 2, Article 12, New York (2016a). http://www. geometrie.tugraz.at/wallner/abw.pdf. Accessed 9 May 2017

Tang, C., Kilian, M., Pottmann, H., Bo, P., Wallner, J.: Analysis and design of curved support structures. In: Adriaenssens, S., et al. (eds.) Advances in Architectural Geometry, pp. 8-22. VDF, Zürich (2016) 